Culture et histoire dans l'espace roman

\title{
Temps et migrations
}

Ibtissam Ouadi-Chouchane

\section{OpenEdition}

\section{Journals}

Édition électronique

URL : https://journals.openedition.org/cher/4640

DOI : $10.4000 /$ cher.4640

ISSN : 2803-5992

\section{Éditeur}

Presses universitaires de Strasbourg

\section{Édition imprimée}

Date de publication : 30 novembre 2021

Pagination : 197-213

ISBN : 979-10-344-0095-9

ISSN : 1968-035X

\section{Référence électronique}

Ibtissam Ouadi-Chouchane, "Temps et migrations », reCHERches [En ligne], 27 | 2021, mis en ligne le 30 novembre 2021, consulté le 12 janvier 2022. URL : http://journals.openedition.org/cher/4640 DOI : https://doi.org/10.4000/cher.4640

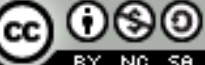

Ce(tte) œuvre est mise à disposition selon les termes de la Licence Creative Commons Attribution Pas d'Utilisation Commerciale - Partage dans les Mêmes Conditions 4.0 International. 


\title{
Temps et migrations
}

\author{
IBTISSAM OUADI-ChOUCHANE
}

L es mouvements migratoires qui ont marqué l'Espagne, autour de la période charnière de la fin des années 1980, ont amorcé une réflexion profonde autour des liens entre les différentes temporalités migratoires et la production théâtrale. Les auteures de notre corpus, Helena Tornero et Irma Correa ont toutes les deux un lien indirect avec les migrants. H. Tornero, résidait enfant dans la gare de Port Bou, un espace de va-et-vient continuels par où de nombreux migrants transitaient. Née d'une mère catalane et d'un père madrilène, la question de l'appartenance identitaire s'est posée depuis son enfance. Quant à Irma Correa, elle a passé son enfance aux îles Canaries où elle est née et où elle a été confrontée quotidiennement au phénomène migratoire. Si cette réflexion identitaire sur l'immigration n'est pas nouvelle, elle prend des formes qui questionnent la réalité à partir de la fiction autour du concept de temps. De la même manière que les migrations, la notion de temps au théâtre est étroitement liée à l'idée de mouvement, de rythme, comme le rappellent les auteurs de l'ouvrage collectif sur le théâtre contemporain espagnol (Amo-Sanchez, Egger, Martinez Thomas, Surbézy, 2005: 53): "Au même titre que l'espace, le temps est une catégorie inhérente au théâtre. Le paramètre temporel est plus étroitement lié aux notions de diachronie, de rythme, de tempo, de mouvement ou de statisme, de passage, de trace, etc.». La réflexion autour du temps historique qui caractérise les mouvements migratoires en Espagne, comme point de départ d'une production littéraire et plus particulièrement théâtrale, est fondamental dans notre corpus. L'objectif de ce travail est de mettre en relation la représentation du migrant au théâtre et son évolution à travers le temps à partir d'une catégorie de migrants souvent oubliés: les enfants. Nous prendrons le terme «enfant» dans sa définition large, en considérant qu'il représente des individus qui n'ont pas encore atteint la majorité, l'âge adulte. L'augmentation du nombre de migrants dans les centres d'accueil pour mineurs en Espagne, entre 2000 et 2019, nous

* Ibtissam Ouadi-Chouchane, Lycée G. de la Tour, à Metz, membre du CHER UR 4376, Université de Strasbourg. 
a amenée à réfléchir à la place de ces jeunes dans la société espagnole et leurs représentations dans la fiction théâtrale. L'expérience de la migration bouleverse ces mineurs qui se retrouvent en perte de repères.

L'analyse de la figure de l'enfant est très intéressante car celui-ci est porteur d'une triple marginalisation: il est étranger, il est rejeté par les adultes car «il est mineur", il se trouve entre deux espaces culturels et ne parvient pas à se définir. Il est également rejeté du groupe en raison de son aspect physique. Ce sont ces problématiques qui sont évoquées dans Sumergirse en el agua et Friday, où l'enfant est le principal protagoniste. Ainsi, le terme d' "altérité», tel que le définit le dictionnaire du Trésor de la Langue Française "caractère, qualité de ce qui est autre, distinct. Antonyme du terme identité», apparaît comme le mieux à même de caractériser les rejets dont font l'objet les protagonistes enfants de notre corpus: exclusion pour des raisons ethniques, identitaires, ou physiques. Il s'agit également de considérer l'importance du contexte historique et économique, et du lien qu'il peut entretenir avec la production dramatique. Le cadre chronologique de notre étude s'étend donc de 2007, publication de Sumergirse en el agua, à 2010, date de publication de Friday. Enfin, les deux pièces oux obtenu le prix SGAE (Sociedad General de Autores de España / Société générale des auteurs d'Espagne) et partagent un processus de création artistique commun: une écriture à partir d'un article de presse. La mise en perspective de ces deux pièces permet d'analyser la représentation de l'altérité enfantine sous des perspectives différentes, (société d'accueil dans la pièce d'Helena Tornero, société de départ dans la pièce d'Irma Correa) et à la fois complémentaires; mais aussi en prenant en compte la différence de destinataires: un public de jeunes adolescents dans le cas de Sumergirse en el agua, et un public adulte dans le cas de Friday. Le traitement réservé au temps dans les deux pièces est très singulier et met en évidence les spécificités propres à l'écriture de chaque auteure. Si dans la pièce d'Helena Tornero, qui se déroule en Europe, les protagonistes ne s'engagent pas dans une longue traversée maritime mais se concentrent davantage sur l'espace de la piscine, comme espace aquatique, symbolique du drame du migrant, la pièce d'Irma Correa, correspond principalement à une succession de temporalités parallèles au centre desquelles se trouvent des jeunes migrants. Deux temporalités se superposent: celle de l'histoire principale du départ de Friday de son village vers l'Europe et celle, statique, de l'attente des jeunes enfants sur le pétrolier.

Ainsi, c'est le lien étroit entre les représentations de l'altérité enfantine sur la scène et la réalité socio-historique espagnole, par le prisme de la fiction, de l'imaginaire que cet article propose d'interroger autour du concept de temporalité. Loin d'un mimétisme total du théâtre à la réalité espagnole et à l'article, il s'agit davantage de comprendre pourquoi les temporalités présentes dans les pièces peuvent constituer un instrument de dénonciation du drame de l'immigration. Nous souhaitons ainsi analyser les œuvres non pas dans un rapport mimétique au monde réel mais comme porteuses du regard de la société sur le monde, tel qu'il est nourri par les médias. En d'autres termes, nous souhaitons examiner la 
figure de l'altérité enfantine et le concept de temps dans deux pièces de théâtre d'auteures espagnoles, ses contours, les stratégies littéraires et dramatiques de sa mise en lumière et en débat; interroger le rôle spécifique qui est donné à cette figure dans la littérature d'une Espagne confrontée depuis les années 1990 à ces mouvements migratoires, qui, comme ailleurs en Europe, ont donné lieu à de nombreux débats, polémiques, résistances et rejets, et surtout de nouvelles lois sur les étrangers (Ley orgánica 4/2000 modifiée par les Lois organiques successives, et toujours plus restrictives, 8/2000, 14/2003, 2/2009, complétées par les décrets sur le Règlement). Le jeu des temporalités à l'œuvre dans les pièces est étroitement lié à la construction de la figure du jeune migrant. Cela nous amènera à réfléchir sur l'impact de cette littérature dans les débats sociétaux et ce que son étude peut apporter en termes d'implication citoyenne.

\section{Temps historique : contextualisation de la thématique migratoire à l'œuvre dans notre corpus}

L’Espagne située dans l'espace méditerranéen a toujours été un espace de rencontres et un véritable creuset de civilisations et de cultures depuis l'Antiquité. Au xx ${ }^{e}$ siècle, l'Espagne franquiste constitue une terre d'émigration. Dès la fin de la guerre civile ce sont plus de 500000 républicains qui quittent le pays lors de la Retirada ${ }^{1}$. Pendant toute la période franquiste de nombreux Espagnols abandonnent le pays pour des motifs politiques, puis économiques. Ils partent vers l'Europe ou l'Amérique Latine. Cette émigration a été fédérée par l'Institut espagnol d'émigration (IEE) de 1956 à 1985. Il s'agit d'un institut créé en 1956 et qui vise à gérer l'émigration d'Espagnols à des fins professionnelles afin de permettre au marché du travail de se réguler, d'avoir comme l'indique Axel Kreienbrink «une soupape de sécurité»:

Tras el plan de aprovechar la emigración, sobre todo hacia Europa, como medio para hacer evolucionar y modernizar a España se ocultaba una lógica semejante a la que ya había sido habitual anteriormente en el Ministerio de Trabajo: liberar el mercado laboral de un excedente de mano de obra, un problema que a finales de los años cincuenta se hizo mucho más urgente al mostrarse el fracaso del modelo económico español basado en la autarquía y al surgir problemas debidos a los esfuerzos por modernizar el país, unos esfuerzos que culminaron con el Plan de Estabilización Económica de 1959. [...] La emigración apareció como posibilidad de lograr la estabilización, haciendo uso de la comparación, a menudo forzada, con una válvula de escape. Por otra parte podía repercutir de forma positiva en la evolución económica, ya que gracias a ella se reducían costes (en concepto de educación, sanidad, gastos sociales) y se obtenían divisas procedentes de las remesas económicas ${ }^{2}$. (Calvo Salgado et al., 2009: 20-21)

1 La Retirada correspond à l'exil de plus de 400000 Espagnols vers la France en 1939 après la victoire du Général Franco.

2 «Derrière le projet de tirer parti de l'émigration, en particulier vers l'Europe, comme moyen de faire évoluer et de moderniser l'Espagne, se cachait une logique 
En 1985, cet institut perd son autonomie et est intégré au Ministère du travail. L'ouvrage collectif de Calvo Salgado, (Calvo Salgado et al., 2009: 21), fait mention des principales destinations des Espagnols: Amérique Latine, France, Allemagne, Royaume-Uni, Belgique, et Suisse. Au total, ce sont près de 2 millions de travailleurs qui ont quitté l'Espagne et qui ont ensuite bénéficié d'une politique de retour. Au début de la transition démocratique, l'Espagne devient une terre d'accueil et une réflexion s'amorce autour d'une série de lois portant sur le statut des étrangers. Ainsi la première loi qui vise à limiter les flux illégaux de migrants a été promulguée en juillet de 1985. Cette loi a été considérée comme très sévère et a fait l'objet d'importantes critiques. Elle est ensuite assouplie en 1987 par le Tribunal Constitutionnel espagnol vers une loi plus progressiste. L'intégration de l'Espagne à la Communauté européenne en 1986, puis à la zone euro en 1999, a fait du pays une des destinations les plus convoitées par les migrants. L'année 2000 marque un tournant important avec plus d'un million d'étrangers. Cette même année est promulguée la réforme de la loi sur le Statut des étrangers, à l'instigation du premier ministre socialiste, qui est la plus progressiste d'Europe en octroyant aux étrangers des droits similaires aux nationaux, comme le précise Rosa M. Soriano-Miras (Soriano-Miras, 2010: 692-693). Cette loi permet une nouveauté: une politique d'intégration. En mars 2000, le conservateur José Maria Aznar remporte les élections et fait de la réforme de la loi sur le statut des étrangers son fer de lance. Il revient à la loi restrictive de 1985 qu'il prend pour modèle. Cette nouvelle loi sera adoptée en 2001. Cependant en mars 2003, treize articles de cette loi seront rejetés par le Tribunal Constitutionnel. La loi sera réformée à deux reprises (septembre, novembre 2003) vers une position plus stricte pour les migrants et de lourdes peines pour les clandestins. La victoire du socialiste José Luis Rodríguez Zapatero, à la tête du gouvernement en 2004, a engendré une nouvelle réflexion quant au statut des étrangers en Espagne. Ce n'est qu'en 2009 que la loi sur le statut des étrangers connaît une profonde réforme avec la modification de soixante articles sur les soixante et onze qui faisaient partie de la loi de 2001. Cette loi aura pour principal axe de travail la nécessaire intégration des étrangers. La chercheuse Rosa M. Soriano-Miras souligne le revers d'une telle situation:

Cabe mencionar como novedad la incorporación a la Ley de nuevos artículos sobre integración en su última reforma, aunque nosotros hemos optado por

pour développer et moderniser l'Espagne, une logique similaire à celle qui était jusqu'alors courante au ministère du Travail: libérer le marché du travail d'un surplus de main-d'œuvre, un problème qui s'était considérablement aggravé à la fin des années 1950 avec l'échec du modèle économique espagnol basé sur l'autarcie et au fur et à mesure que des problèmes surgissaient en raison des efforts de modernisation du pays, qui aboutissent au Plan de Stabilisation Économique de 1959. [...] L'émigration est apparue comme une possibilité de parvenir à la stabilisation, en utilisant la comparaison souvent forcée, avec une valve d'échappement. D'autre part, l'émigration pourrait avoir des répercussions positives sur le développement économique, car elle réduisait les coûts (éducation, santé, sécurité sociale, etc.) et on obtenait des devises étrangères provenant des transferts de fonds économiques.» 
denominar este giro como integración coercitiva, pues, lejos de promover dicha integración a través de la convivencia, se presenta con un cierto carácter impositivo ${ }^{3}$. (Soriano-Miras, $2010: 696$ )

La réforme proposée en 2009 permet d'intégrer de nombreuses avancées sociales: l'amélioration de l'accès au marché du travail pour les étrangers, l'autorisation du droit de réunions pour les immigrés en situation illégale, un droit d'accès à l'éducation pour les clandestins jusque 18 ans, une meilleure gestion des mineurs étrangers non accompagnés (MENA), un élargissement du regroupement familial aux couples non mariés et une protection renforcée des femmes contre la violence domestique. Néanmoins l'intégration prend un caractère imposé. Cette réforme de la loi a été très mal perçue par les partis conservateurs qui la considèrent comme l'une des causes de l'augmentation de l'immigration illégale. Les MENA sont des mineurs qui se retrouvent seuls en Espagne. Leur nombre ne cesse de croître et en 2018, les autorités européennes ont comptabilisé une augmentation de 405\% de leur nombre (Caro. L, 2019a). Ils sont souvent orphelins et tentent l'aventure vers l'Europe. Certains perdent leurs parents durant la traversée. Ces mineurs sont principalement originaires d'Afrique comme le précise le rapport du procureur général, pour l'année 2017:

El $97,05 \%$ (2.276) son de sexo masculino, y el resto (69) son niñas. Mayoritariamente proceden de Marruecos (56,33\%), Argelia (19,95\%) seguidos por países del África Subsahariana como la República de Guinea (8,48\%) Costa de Marfil (7,50\%) y Gambia (2,85\%). Al igual que en otros años no se disponen de datos cuantitativos sobre los menores que se han introducido en España a través de Ceuta y Melilla, superando la frontera ocultos en vehículo de motor o por otras vías, como paso previo a embarcarse como polizones en buques con dirección a la Península4. (Fiscal.es, 2018)

Le jeune nigérien, Friday T., qui a inspiré l'écriture de la pièce Friday à Irma Correa fait partie de ces mineurs non accompagnés. Nous reviendrons sur cet aspect dans l'analyse de la pièce.

3 «Il convient de mentionner comme nouveauté l'incorporation dans la loi de nouveaux articles sur l'intégration dans sa dernière réforme, bien que nous ayons choisi de qualifier ce changement d'intégration coercitive, puisque, loin de favoriser l'intégration par la coexistence, elle présente des aspects contraignants."

$4 \ll 97,05 \%$ (2276) sont des garçons, et le reste (69) sont des filles. La majorité d'entre eux proviennent du Maroc (56,33\%), de l'Algérie (19,95\%), suivis de pays d'Afrique subsaharienne tels que la République de Guinée (8,48 \%), la Côte d'Ivoire $(7,50 \%)$ et la Gambie (2,85\%). Comme pour les années précédentes, aucune donnée quantitative n'est disponible sur le nombre de mineurs entrés en Espagne via Ceuta et Melilla, en franchissant la frontière cachés dans un véhicule à moteur ou par d'autres moyens, première étape avant de embarquer en tant que passagers clandestins sur des navires à destination de la péninsule ibérique.» 


\section{Du temps historique à la pièce}

Helena Tornero est une artiste polyvalente: dramaturge, scénariste, metteur en scène, enseignante et traductrice, elle a beaucoup travaillé ces dernières années entre la France et l'Espagne. Durant son enfance, elle cultive sa passion pour le théâtre. Néanmoins, elle poursuit des études de tourisme à l'Université de Gérone et obtient un poste confortable. Après dix ans de carrière, elle décide subitement de donner un nouveau tournant à sa vie et de se lancer à l'âge de 30 ans dans une carrière théâtrale après le succès de sa première pièce qui reçoit le prix Joan Santamaria. Le sujet de cette pièce, El vals de la garrafa, 2003, est inspiré d'une histoire vraie: celle de son grand-père Emilio Brugués durant la période franquiste. Helena Tornero s'engage dans des études théâtrales, étudie la mise en scène et la dramaturgie à l'Institut de Teatre de Barcelone. En 2007, elle est récompensée par le prix SGAE pour Sumergirse en el agua. Cette pièce met en scène des adolescents dont un jeune migrant de deuxième génération: Josué. La violence et la frustration de jeunes adolescents (Thomas et Stefan) débouche sur un drame: la mort de Josué. Dans cette pièce, l'auteure souligne l'indifférence et le manque de prise de position face au rejet de l'autre.

Quant à Irma Correa, originaire des îles Canaries, elle a très tôt été confrontée au phénomène de l'immigration. Diplômée de droit, de journalisme et d'études théâtrales - Real Escuela Superior de Arte Dramático (RESAD) 5 - (2001-2006), elle se définit comme une artiste polyvalente: actrice, auteure, metteur en scène. Elle obtient le prix SGAE en 2010 pour Friday. Cette pièce, qui s'inspire d'un article de presse, met en scène un jeune nigérien, Friday, qui rêve de devenir footballeur professionnel. Après avoir quitté son village natal, Friday et son frère Olusegun s'orientent vers une ville portuaire afin de tenter l'aventure de l'émigration. La fin tragique de la pièce, qui se traduit par la mort de Friday, dénonce la violence et les épreuves que subissent les jeunes migrants tout au long de leur périple vers l'Europe.

C'est à partir de la lecture d'articles dans la presse qu'Irma Correa et Helena Tornero ont décidé d'écrire leurs pièces. C'est justement de ce lien entre la société et le théâtre que nous sommes partis pour analyser notre corpus; lien que la chercheuse américaine Eileen Doll souligne dans son étude sur la dramaturgie espagnole et le phénomène migratoire "Como el teatro muchas veces sirve de espejo o termómetro de la sociedad, ya era hora de indagar en el escenario en el tema de la inmigración y sus consecuencias ${ }^{6}$ (Doll, 2013: 20)». Le premier article qui a retenu l'attention d'Helena Tornero et qui lui a servi de point de départ

5 La RESAD : il s'agit d'une prestigieuse institution qui se trouve à Madrid et qui a été inaugurée au XIX ${ }^{\mathrm{e}}$ siècle. Elle propose un enseignement artistique supérieur théâtral. De nombreux auteurs contemporains se sont formés dans cette institution, nous reviendrons sur ce point dans le chapitre 2 de la première partie.

6 "Comme le théâtre sert souvent de miroir ou de thermomètre de la société, il était grand temps d'investiguer sur scène la question de l'immigration et ses conséquences.» 
dans son processus d'écriture est un article d'Eusebio Val publié dans le journal El País qui relate le meurtre d'un enfant de six ans, à la piscine municipale, en Allemagne, commandité par un néonazi (Val, 2001). La photo qui illustrait l'article a fortement marqué l'auteure en raison de la violence perceptible chez les jeunes nazis. Un autre article sur un fait divers espagnol a inspiré l'écriture de Sumergirse en el agua. Une fois de plus les principaux acteurs du drame sont des jeunes. Il s'agit d'une sans abri qui dormait à proximité d'un distributeur automatique et qui a été brûlée vive par ces jeunes. Ce drame a suscité beaucoup d'émoi au sein de l'opinion publique et d'autres auteurs comme Paloma Pedrero ${ }^{7}$ qui a écrit une pièce sur le sujet. C'est la violence gratuite à l'œuvre dans ce drame qui sert de soubassement à la pièce de l'auteure catalane. Ce sont ces deux articles et les photos associées qui vont inciter l'auteure à dénoncer la passivité et l'indifférence des gens face à ces drames. Pour ce qui est de la pièce d'Irma Correa, le regard du jeune enfant a beaucoup marqué l'auteure. Cette dernière le précise dans un article du journal El País:

«Tenía una mirada tan triste», recuerda la autora. «Su mirada y las razones que le llevaron a subirse a ese petrolero hicieron que yo quisiera escribir su historia», dice. La actriz y dramaturga subraya que hablar del drama de la inmigración infantil es una manera de hablar del de la adulta que, desde África, idealiza el viaje a Europa para conseguir una vida mejor, tener un trabajo, ganar dinero, y mantener a su familia, pero visto «desde un punto de vista mucho más puro», añade ${ }^{8}$. (García Saleh, 2010)

C'est ce regard si singulier du jeune Friday T, fraîchement arrivé aux Canaries, qui est décrit par le journaliste Juan Manuel Pardellas, auteur de l'article qui a inspiré Irma Correa (Pardellas, 2007):

$\mathrm{Al}$ despedirse, su mirada y sus labios aún recuerdan el niño que es. Se vuelve a poner la gorra, agacha la cabeza y regresa a la sala de vídeo donde proyectan $E l$ circo del Sol .

Ce regard est porteur des épreuves que le jeune nigérien a connues et qui l'ont poussé à grandir précipitamment comme c'est le cas dans la pièce. La singularité de la pièce est de mêler la dimension politique et poétique. Les deux

7 Il s'agit de la pièce Caídos del cielo, 2008 de Paloma Pedrero. Dans cette pièce, P. Pedrero met en scène l'histoire d'une sans abri brûlée vive par trois jeunes dans le hall d'un distributeur automatique. Pour lire la pièce cf. <http://www.contextoteatral.es/ caidosdelcielo.html>, consulté le 05.02.2020.

8 "Il avait un regard si triste", se souvient l'auteur. "Son regard et les raisons qui l'ont poussé à monter sur ce camion-citerne m'ont donné envie d'écrire son histoire", dit-elle. L'actrice et dramaturge souligne que parler du drame de l'immigration des enfants est une façon de parler des adultes qui, depuis l'Afrique, idéalisent le voyage vers l'Europe pour avoir une vie meilleure, avoir un travail, gagner de l'argent et aider leur famille, mais vu "d'un point de vue beaucoup plus pur", ajoute-t-elle.»

9 «Lorsqu'il dit au revoir, son regard et ses lèvres rappellent encore l'enfant qu'il est. Il remet sa casquette, incline la tête et retourne dans la salle vidéo où la salle vidéo où est projeté le Cirque du Soleil.» 
espaces/temps et les deux histoires s'entrecroisent pour déboucher sur la mort de Friday enfant, seul le Friday devenu adulte survit.

\section{Un temps dramatique caractérisé par la superposition}

Dans les deux pièces, des phénomènes de superposition s'opèrent. Deux temporalités se superposent dans Friday: celle de l'histoire principale du départ de Friday de son village vers l'Europe et celle statique de l'attente des jeunes enfants sur le pétrolier. Dans cette pièce, la mise en place de deux espaces temps concomitants permet de passer progressivement d'un temps dramatique à un temps mythique par le biais de la musicalité du djembé; puis à un temps magique, propre à l'univers de l'enfance. C'est cet aspect qui est perceptible dans l'échange avec Gabriel, un jeune enfant plus âgé que Friday:

FrIDAY.- ¿ Juegas al fútbol?

Gabriel. - ¿Yo? No.

Friday.- Yo, sí. Y voy a ser como Messi.

EmeкA.- Más te valdría quitarte esas cosas de la cabeza.

Gabriel. - ¿ Por qué no como Eto’o? ¿O como Kanú, el más grande que ha dado Nigeria?

Friday.- Porque ellos no vuelan (Correa, 2011: 37-38) ${ }^{10}$.

Gabriel souligne également par sa remarque l'existence de footballeurs africains célèbres mais Friday ne peut pas les idéaliser autant que Messi car ils sont Africains, ils appartiennent à la même réalité dont il est issu, car «ils ne volent pas». C'est, dans un premier temps, au travers de l'espace onirique que Friday se construit une image de l'Europe (Correa, 2011: 69)

FRIDAY: Él está ahí en medio de los jugadores, rodeado. No le tiembla ni un músculo. Espera. Empieza a correr. Corre, corre, corre con el balón entre sus pies, y es como un espíritu, porque todo lo que se le pone delante lo atraviesa. Es como si no tuviera cuerpo. Como si pudiera ir a cualquier parte porque nada ni nadie se lo impide. Es como un suspiro de madre antes de morir ${ }^{11}$.

À mesure que le récit avance, Friday, proche de la mort, passe du rêve à l'hallucination puis de l'illumination à la mort. Il devient alors le joueur de football européen qu'il décrivait à la scène 16, passant ainsi de la troisième personne du singulier à la première personne du singulier (Correa, 2011: 87):

10 Friday. - Tu joues au football? / Gabriel.- Moi? Non. / Friday.- Moi oui, et je vais être comme Messi./Emeka.- Tu ferais mieux de te sortir ces choses de la tête./ Gabriel.- Pourquoi pas comme Eto'o? ou comme Kanu, le plus grand Nigérian de tous les temps? / FridaY.- Parce qu'ils ne volent pas.

11 Friday.- Il est là, au milieu des joueurs, entouré. Il ne bouge pas d'un poil. Il attend. Il commence à courir. Il court, court, court avec le ballon entre les pieds, et il est comme un esprit, parce que tout ce qui vient devant lui passe par lui. C'est comme s'il n'avait pas de corps. Comme s'il pouvait aller n'importe où parce que rien ni personne ne l'arrête. C'est comme le soupir d'une mère avant sa mort. 
Friday.- Y estoy ahí en medio, rodeado. No me tiembla ni un músculo. Espero. Y empiezo a correr. Corro, corro, corro con el balón entre mis pies, y soy como un espíritu, porque todo lo que se me pone delante lo atravieso. Es como si no tuviera cuerpo. Como si pudiera ir a cualquier parte, porque nada ni nadie me lo impide. Soy como un suspiro de madre antes de morir.

Fin (y principio) ${ }^{12}$

Friday est libéré de toute corporalité, de toute contrainte et s'éteint, comme sa mère au début de la pièce. Les images qui se dégagent des répliques de Friday lui permettent de glisser dans un espace poétique singulier qui se construit tout au long de son périple sur le bateau. Le passage du temps dramatique à un temps mythique, magique se fait par le biais du rêve. À partir de la scène 14, Friday souffre d'une fièvre importante et a des visions hallucinatoires, Emeka tente d'y remédier (Correa, 2011: 64):

Emeka pone las manos sobre la frente de Friday. Reza por lo bajo. Hace aspavientos. Comienza un ritual grotesco.

GABRIEL.- Creo que con eso bastará.

EMEKA.- ¡No he terminado!

GABriel. - Bastará. Seguro.

Friday (Señalando al cielo) Es una mariposa. Una mariposa.

Friday se levanta, señalando aún. Gabriel y Emeka salen, y dirigen la mirada hacia el punto que señala Friday. Entran Gani y Maliki cogen la caja de frutas ${ }^{13}$.

Friday indique voir des papillons. Or il s'agit d'une image qui fait écho aux visions de sa mère avant de mourir à la scène 7 (Correa, 2010: 36).

Cette image du papillon, un animal libre et volant, est ensuite complétée par un univers visuel poétique qui se clôturera à la mort de Friday (Correa, 2011:84-85):

FrIDAY : Hay una pequeña luz que aparece y desaparece, aparece y desaparece, aparece y desaparece.

La pelota de trapo se escapa del regazo de Friday. Este se levanta y va tras ella. Oscuro. Un foco ilumina a Friday. Gabriel grita su nombre, a lo lejos, mientras suena la sirena del barco. En el fondo del escenario aparece la luz de un faro ${ }^{14}$.

12 «FridAY._Et je suis là, au milieu, encerclé. Je ne secoue pas un muscle. J’attends. Et je commence à courir. Je cours, je cours, je cours avec le ballon entre les pieds, et je suis comme un esprit, parce que tout ce qui est devant moi, je le traverse. C'est comme si je n'avais pas. Comme si je pouvais aller n'importe où, car rien ni personne ne peut m'arrêter. Je suis comme le soupir d'une mère avant sa mort. / Fin (et début)»

13 «Emeka pose ses mains sur le front de Friday. Il prie tout bas. Il gesticule. Il commence un rituel grotesque. / GABRIEL.- Je pense que ça devrait suffire. / EMEKA. - Je n'ai pas fini! / Gabriel. - Ça suffira. J'en suis sûr. / Friday. - (montrant le ciel) C'est un papillon. Un papillon. / Friday se lève, toujours en pointant du doigt. Gabriel et Emeka sortent, et lèvent les yeux vers le point que Friday désigne. Gani et Maliki entrent et prennent la boîte de fruits.»

14 «FRIDAY.- Il y a une petite lumière qui apparaît et disparaît, apparaît et disparaît, apparaît et disparaît. / La balle de chiffon glisse du genou de Friday. Il se lève et va la chercher. / Sombre. Une lumière éclaire Friday. Gabriel crie son nom, au loin, alors 
La lumière - métaphore de la vie - semble fragile à l'image de la situation critique de Friday, qui, totalement déshydraté, peine à se maintenir debout. L'itération des verbes "aparece y desaparece» semble scander une mort proche. Friday séteint mais sa lumière, son rêve ne séteignent pas avec la mort.

\section{Chez Tornero la superposition des temporalités se traduit par une superposition des voix}

La superposition des temporalités engendre une confusion des espaces entre la réalité et la fiction qui correspond à la problématique clé de la pièce. La scène 9 illustre clairement cette situation. En effet, la macrodidascalie aperturale donne des indications précises qui permettent de comprendre le sens du jeu scénique (Tornero, $2008: 77$ ):

Dos espacios diferenciados, uno al lado del otro, separados por una pared y una puerta, visibles o invisibles. Se trata de una comisaría de policía. En el primer espacio se encuentran María y el inspector GEORG. En el segundo espacio, tres policías están revisando y clasificando formularios, pero toda su atención la centran en el juego de adivinar películas, juego que suspenden cada vez que creen que el inspector los va a pillar in fraganti ${ }^{15}$.

La didascalie décrit deux espaces distincts dont la frontière est matérialisée par une porte qui permet d'être vu ou non. On retrouve ici le jeu du voir/sans être vu dans une double mise en abîme. Les acteurs sont observés par le public. Les policiers observent, María et l'inspecteur, et jouent à imiter ce dernier (Tornero, 2008: 79):

Georg se sitúa delante de María, expectante. El Policía 3 hace los gestos de un hombre que observa y escucha atentamente a alguien. Sus gestos podrían coincidir unos segundos con los del inspector. MARÍA mira al inspector GeORG y calla ${ }^{16}$.

Toute une communication non verbale se met en place. La métathéâtralité de la pièce engendre une confusion des voix (Tornero, 2008: 78): «GEORG/ Policía: (A la vez) Pensar es sano»(Georg/ Police: (en même temps) Penser est sain D’un côté, Georg et María parlent de la réalité et du drame de la mort de Josué. De l'autre côté, les policiers parlent de fiction et jouent à deviner des titres de films. Tout au long du dialogue un enchâssement se met en place et engendre une confusion de deux temporalités: réalité/fiction. Georg indique à

que la sirène du bateau retentit. En arrière-plan de la scène, la lumière d'un phare apparaît.»

15 «Deux espaces distincts, côte à côte, séparés par un mur et une porte, visibles ou non. C'est un poste de police. Dans le premier espace se trouvent Maria et l'inspecteur Georg. Dans le deuxième espace, trois policiers vérifient et classent des formulaires, mais toute leur attention est concentrée sur un jeu pour deviner des titres de films qu'ils arrêtent à chaque fois qu'ils pensent que l'inspecteur va les surprendre.»

16 «Georg se tient devant Maria, dans l'expectative. Le Policier 3 fait les gestes d'un homme qui regarde et écoute attentivement quelqu'un. Ses gestes pourraient coïncider avec ceux de l'inspecteur pendant quelques secondes. Maria regarde l'inspecteur GEORG et se tait.» 
María que son hypothèse - celle d'une mort préméditée - n'est que pure fiction, (Tornero, 2007: 80) Ce jeu est observé par les policiers qui tels des spectateurs vont commenter la scène comme s'il s'agissait d'un film (Tornero, 2008: 80-81):

El inspector GEORG aplaude. Los policías miran hacia la puerta.

Policía 1.- ¿Qué hace?

Policía 2.- Tonterías. No sé a qué viene tanta comedia. Lleva horas así. El caso está cerrado y bien cerrado. Esa mujer no tiene nada que hacer. Es evidente que fue un accidente. Lo que debe querer es sacar pelas.

Policía 1.- ¿Y tú cómo sabes que fue un accidente?

Policía 2.- Intuición, tío, intuición. ¿No dicen todos que no vieron nada? Pues si dicen que no vieron nada, es que no pasó nada. Es evidente, tío. La gente no quiere problemas. No, si yo fuera el inspector, ya me habría quitado de encima a esa mujer. Va, Peter, continúa ${ }^{17}$.

L'inspecteur applaudit María comme s'il venait d'assister à un spectacle lui ôtant ainsi toute crédibilité. Il est alors spectateur de María, observé par les policiers. Enfin, le public observe l'intégralité de la scène. Ce système d'enchâssement permet de mettre en scène la problématique du voir/sans voir.

\section{Le temps dramatique est marqué par un rythme singulier}

Du point de vue structurel la pièce d'Irma Correa se construit avec un rythme qui est perceptible à travers les répétions de scènes: la scène 2 fait écho à la scène 12. Il s'agit de scènes qui situent l'action sur l'espace symbolique du pétrolier, espace intermédiaire entre l'Afrique et l'Europe et qui sous-tendent la pièce. Ces répétitions structurelles qui scandent le temps dramatique sont également caractérisées par des transitions. Le ballon de football sert de transition entre les scènes. Tel un fil d'Ariane il permet le passage d'une scène à l'autre. Son état évolue à l'image du personnage de Friday. Des jeux de rythmes sont à l'œuvre à la scène 18 (accélération du rythme à mesure que le danger approche), scène de violence qui débouche sur un drame: Friday poignarde Oso, nous y reviendrons.

17 «L'inspecteur Georg applaudit. Les policiers regardent vers la porte. / Police 1.Que fait-il? / Police 2.- N'importe quoi. Je ne sais pas à quoi rime cette comédie. Ça fait des heures que ça dure. L'affaire est close et bien close. Cette femme n'a rien à faire. Il est évident que que c'était un accident. Ce qu'elle doit vouloir, c'est se faire de l'argent. / Police 1.- Et comment sais-tu que c'était un accident? / Police 2.Intuition, mec, intuition. Ne disent-ils pas tous qu'ils n'ont rien vu ? Bien, s'ils disent qu'ils n'ont rien vu, alors il ne s'est rien passé. C'est évident, mec. Les gens ne les gens ne veulent pas de problèmes. Non, si j'étais l'inspecteur, je me serais déjà débarrassé de cette femme. / Vas-y, Peter, continue.» 


\section{Le jeu de rythmes sert également la dénonciation de ce que Helena Tornero appelle le "pacte du silence»}

Dans Sumergirse en el agua, la scène 7, qui se trouve presqu'au centre de la pièce, évoque l'importance du regard, de l'image. La structure temporelle de cette scène est très intéressante car elle met en évidence graduellement la construction du stratagème des spectateurs de la mort de Josué qui décident de "fermer les yeux» et de déclarer, d'un commun accord, ne rien avoir vu. Le premier mouvement commence par la constatation de la forte fréquentation de la piscine au travers d'une gradation quantitative désignée par le terme "otro» (Tornero, 2008: 103):

OJOS (Uno, Dos, Tres, Cuatro, todos ante el público. Rápido, con agilidad. A veces unos hacen eco de lo que dicen los otros.)

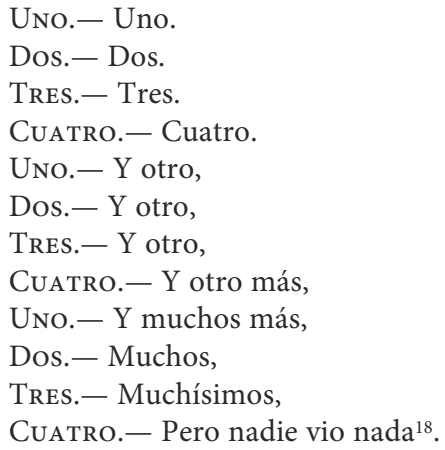

La didascalie aperturale souligne la présence des personnages l'un à côté de l'autre face au public comme s'il s'agissait de la comparution de coupables. C'est ce que suggère la lecture proposée de la pièce à la Mousson d'hiver en $2018^{19}$. Les personnages sont face aux spectateurs comme le suggère la didascalie. L'alignement des personnages derrière une ligne matérialisée par du ruban adhésif et l'éclairage du fond de la scène font penser à une scène d'identification des coupables dans un poste de police. Cette scène fait écho à la scène finale de la mort de Josué où on assiste de la même manière à la comparution de tous les personnages. Dans ce passage, la structure rythmique répétitive de la scène met en évidence le souhait pour les quatre personnages - désignés chacun par un chiffre - de se convaincre de la nécessité de mentir. La gradation quantitative de témoins est interrompue par la conjonction de coordination "pero» qui

18 «OJOS (Un, Deux, Trois, Quatre, tous devant le public. Rapidement, avec agilité. Parfois les uns font écho à ce que disent les autres). / Uno.- Un. / Dos.- Deux. / Tres. - Trois / Cuatro.- Quatre. / Uno.- Et un autre, / Dos. - Et un autre, / Tres. - Et un autre, / Cuatro. - Et un autre, / Uno. - Et beaucoup d'autres, / Dos.- Beaucoup, / Tres. - Très nombreux, / CuAtro.- Mais personne n'a rien vu. »

19 Mousson d'hiver 2018, en collaboration avec le Théâtre de la Manufacture, Photos Eric Didym, Lecture dirigée par Christine Koetzel, avec les étudiants L3 Études culturelles (Nancy), <https://www.meec.org/mousson-dhiver-2018>, consulté le 02.02.2020. 
interpelle le spectalecteur. En effet, en dépit du nombre important d'individus dans l'eau personne n'a rien vu. C'est d'ailleurs, cette contradiction qui amène Maria, la mère de Josué, à douter quant au statut accidentel de la mort de son fils. Commence ensuite la deuxième phase de l'argumentation: le déni. Du déni personnel (Tornero, 2008: 62) «yo no vi nada» (moi, je n’ai rien vu) au déni collectif clamé en cœur «todos: no vimos nada» (tous: nous n'avons rien vu), qui est ensuite renforcé par des questions rhétoriques "¿Qué chicos?» (quels enfants?) puis diverses formes de généralisations afin de renverser la situation. En effet, les témoins/menteurs accusent les autres de mentir. Ce sont les autres qui tiennent des propos mensongers (Tornero, 2008: 63) «Dicen mentiras. La gente dice mentiras» (Ils disent mensonges. Les gens disent des mensonges). La troisième étape est caractérisée par ce que H. Tornero appelle le «pacte du silence» (Tornero, 2008: 64-65):

Dos.- ¡Ah! (Pausa.) ¡Ah, sí! (Pausa.) Pues no. Yo no las vi. Yo tampoco las vi. (A los demás.) No vi nada.

Uno.- (Al público.) No vio nada.

Todos. - Nada de nada ${ }^{20}$.

Progressivement le discours de «Dos» est rapporté par «uno» puis par l'intégralité du groupe donnant ainsi plus de force au propos. La rupture du quatrième mur, perceptible à travers la mésodidascalie "al público», marque un moment important dans ce stratagème mensonger. Il s'agit de rallier le spectalecteur au point de vue des témoins, de convaincre le spectalecteur de la nécessité et du bien-fondé du mensonge. Cependant, les mésodidascalies «(Pausa)» imposent un silence gênant et dérangeant. Dans la pièce l'utilisation de pause ou silence est fréquente. Ces mésodidascalies mettent en exergue des passages importants ou imposent le silence. Un silence qui est porteur, c'est le cas ici, d'une certaine culpabilité. Patrice Pavis, dans son Dictionnaire $d u$ théâtre, consacre précisément une section spécifique à la notion de silence. Le sémiologue propose dans son dictionnaire une typologie des silences au théâtre. Il distingue quatre types différents de silence: le silence déchiffrable, le silence d'aliénation, le silence métaphysique et le silence bavard. Le silence présent dans Surmergirse en el agua semble correspondre à ce que P. Pavis appelle le silence déchiffrable (Pavis, 2004: 326-327):

C'est le silence psychologique de la parole refoulée; par exemple: STRINDBERG, TCHEKHOV, aujourd'hui le VINAVER du Théâtre de Chambre. On perçoit assez bien ce que le personnage refuse de révéler, et la pièce est fondée sur cette dichotomie entre non-dit et déchiffrable; le «sens» du texte est de savoir fonder l'opposition entre dit et non-dit.

20 «Dos.-Ah! (Pause.) Ah, oui! (Pause.) Eh bien, non. Je ne les ai pas vus. Je ne les ai pas vus non plus. / (Aux autres.) Je n'ai rien vu. / Un.- (Au public.) Il n'a rien vu. / Tous.- Rien du tout.» 
Quant à Sanda Golopentia (Golopentia, 1994), elle s'est également intéressée au silence au théâtre et a souligné une autre typologie celle de Bernard Dauenhauer, que nous pouvons appliquer à notre extrait. En effet, B. Dauenhauer, pour sa part, distingue trois types de silences. D'abord le «fore and after silence» que Sanda Golopentia appelle «silence-cadre» puisqu'il apparaît avant et après avoir pris la parole. Le deuxième type de silence évoqué par le chercheur est le «intervening silence» que Sanda Golopentia appelle le «silence-intercalaire»: sa fonction est d'assurer la délimitation des mots et des réponses. C'est précisément le processus qui se produit dans le passage que nous avons étudié et qui correspond à la concertation des différents témoins. Le dernier silence évoqué par l'auteur est le "deep silence»: un silence qui souligne l'importance de ce qui va se passer. C'est ce silence qui est marqué par la mésodidascalie «pausa» à la fin de la scène 7 (Tornero, 2008: 65): «Dos: Entonces no pasó nada. (Pausa) ¿No pasó nada, verdad?» (Dos: Alors rien ne s'est passé. Il ne s’est rien passé. (Pause.) Il ne s'est rien passé, n'est-ce pas?). Le personnage de «dos» est tiraillé entre le poids du groupe et un mensonge qui lui pèse. Après cette réplique, les répliques s'enchaînent à profusion comme pour cacher le mal-être suggéré par ce silence. Enfin, la scène se termine par un passage très fort qui met en évidence la frustration des personnages (en raison du mensonge collectif imposé) et qui débouche sur une violence verbale (Tornero, 2008: 68-69):

Cada vez más rápido, más exagerado y con más volumen.

Dos. - ¡Yo no he visto nada!

Tres. - ¡Yo no he visto nada!

Cuatro.- ¡YYo no he visto nada!

Más rápido, gritando.

Uno.- ¡Yo no he visto nada!

Dos._ ¡Yo no he visto nada!

Tres. - ¡Yo no he visto nada!

Cuatro : ¡Yo no he visto nada!

Uno.— ¡Nada!

Dos. — ¡Nada!

Tres. - ¡Nada!

Cuatro.— ¡Nada!

Oscuro. Sonido de un cuerpo que cae al agua. Oímos la voz de UNO, en la oscuridad.

UNO : ¿Lo veis? ¡Más claro, el agua!21

21 «De plus en plus vite, de plus en plus exagéré et avec de plus en plus de volume. / Dos. - je n'ai rien vu! / Tres. - Je n'ai rien vu! / Cuatro.- Je n'ai rien vu! / Plus vite, en criant. / Uno. - je n'ai rien vu! / Dos. - je n'ai rien vu! / Tres. - Je n'ai rien vu! / Cuatro.- je n'ai rien vu! / Uno.- Rien! / Dos.- Rien! / Tres.-Rien! / Cuatro.- Rien! / Sombre. Bruit d'un corps tombant dans l'eau. Nous entendons la voix de Uno, dans l'obscurité. / Uno.—-Tu vois, plus clair, l'eau!» 
Les personnages psalmodient un à un le discours commun à tenir face à la mort de Josué. La violence est exacerbée par le rythme - «más rápido» - mais aussi par le volume sonore crescendo des voix des personnages. Il s'agit de crier très fort le pacte du silence. Ce paradoxe est souligné par la dernière didascalie et réplique de la scène (Tornero, 2008: 69): «Oscuro. Sonido de un cuerpo que cae al agua. Oímos la voz de UNO, en la oscuridad. UNO: ¿Lo veis? ¡Más claro, el agua!». L'ironie qui naît du jeu de mot autour de l'eau et l'action de voir insiste sur la confusion et l'aspect trouble de la situation. Le personnage de « uno» nous invite à voir clairement, alors que la scène est totalement plongée dans le noir «oscuro»- une absence de visibilité qui s'oppose à une présence sonore de corps qui tombent à l'eau. La contradiction entre la vue et l'ouie souligne l'obscurité de la situation. C'est cette problématique qui est au cœur de la scène 11. En effet, les mésodidascalies qui encadrent la scène (Tornero, 2008: 89): «Sonido de un cuerpo que cae al agua» sous-entendent la présence d'individus dans la piscine, espace où a eu lieu le meurtre prémédité de Josué. Ainsi Sara met en lumière la difficulté à oublier le drame en raison de la multitude de témoins (Tornero, 2008: 105):

SARA: Sería mucho más fácil de olvidar si la piscina hubiese estado un poco más vacía. Si no hubiese habido tanta gente allí, mirándolo todo, habría sido mucho más fácil de olvidar» ${ }^{22}$.

Elle dénonce deux problèmes : le meurtre de José et l'attitude fourbe des témoins qui préfèrent s'enfermer dans le mensonge et le silence.

Chez Irma Correa, l'accélération du rythme est également perceptible au moment de signaler les abus dont souffrent les jeunes migrants. Ces derniers sont d'abord des victimes de la violence de leur oncle, qui, à la suite du décès de leur mère, souhaite les envoyer récolter du cacao dans un autre village (Correa, 2010: 42). Olusegun souffre ensuite de la violence de Funto, qui souhaite l'exploiter économiquement (Correa, 2010: 68). Le dernier épisode de violence auquel assiste Friday est celui qui a lieu sur le bateau entre Olusegun et Oso (Correa, 2010: 81):

Oso coge la mano de Olusegun y se la pone en su muslo. Suena el djembé furioso $y$ gritos de cavernas. Las luces bajan de intensidad al tiempo que los personajes se mueven con lentitud. Olusegun muerde en la cara a Oso. Ojkwu Y Gani se enzarzan. Violencia, sangre, caos. [...]

Friday.- Olusegun (Se levanta) Y me levanté, despacio, (coge la navaja de Ojkwu) y cogí el cuchillo que estaba en el suelo, y lo hundí varias veces en el señor gordo. (Tira la navaja, coge la pelota) Y salí. Y cerré la puerta sin hacer ruido. Y fue como si, de repente, me hubiera hecho mayor $^{23}$.

22 «SARA: Ce serait beaucoup plus facile d'oublier si la piscine avait été un peu plus vide. S'il n'y avait pas eu autant de personnes présentes, regardant tout, il aurait été beaucoup plus facile d'oublier.»

23 «Oso prend la main d'Olusegun et la pose sur sa cuisse. On entend des djembés furieux et des cris caverneux. Les lumières se tamisent alors que les personnages se déplacent lentement. Olusegun mord Oso au visage. Ojkwu et Gani se battent à coups 
Friday, témoin à deux reprises de la violence des adultes envers les enfants, bascule et devient à son tour violent et adulte en poignardant Oso.

\section{Conclusion}

Ainsi, les deux auteures de notre corpus sont parties de la réalité sociohistorique espagnole pour modeler leur écriture et exprimer leur engagement poétique et artistique. Les réflexions autour des articles et des pièces de notre corpus nous ont amenée à étudier en détail le rôle et l'importance des temporalités. Si Irma Correa fait le choix du jeu rythmique et poétique autour du temps historique, mythique et magique; Helena Tornero exploite quant à elle les rythmes et les effets de superpositions temporelles pour mieux dénoncer le drame de l'immigration. La distinction de perceptions des temporalités, temps historique/ temps du récit, menée par Marcel Vuillaume, dans le cadre d'une réflexion sur le roman, pourrait tout à fait s'appliquer au théâtre et à nos œuvres (Vuillaume, 2019):

[...] la curiosité que suscite un événement du monde réel, relaté, par exemple, dans un ouvrage historique, n'est pas la même, et ne s'exprime pas de la même façon, que la curiosité qu'éveillent les faits racontés dans un roman. Dans le premier cas, on veut savoirce qui s'est passé, alors que, dans le second, on veut savoir ce qui va se passer. Nous vivons au présent les événements dont nous lisons le récit et nous concevons comme futurs ceux dont nous n'avons pas encore pris connaissance.

C'est cette distance temporelle qui est au cœur de l'engagement des auteures pour dénoncer le drame de l'immigration et l'indifférence de la société.

\section{Bibliographie}

Amo-Sanchez A., Egger, C., Martinez Thomas, M., Surbézy, A., 2005, Le théâtre contemporain espagnol, approche méthodologique et analyse de textes, Rennes, Presses Universitaires de Rennes.

Calvo Salgado et al., 2009, Historia del Instituto Español de Emigración. La política migratoria exterior de España y el IEE del franquismo a la transición, Madrid, Ministerio de Trabajo e Inmigración.

Caro. L, 2019a, «La UE cifra en un 405\% el aumento de menores inmigrantes en España en 2018», ABC.ES, 13/03/2019, < https://www.abc.es/espana/abci-cifra405-por-ciento-aumento-menores-inmigrantes-espana-2018-201903170253_

de poing. Violence, sang, chaos. [...] / Friday._- Olusegun (se lève) Et je me suis levé, lentement, (ramasse le couteau d'Ojkwu) et j'ai pris le couteau qui était par terre, et je l'ai plongé plusieurs fois dans le gros homme. (Jette le couteau, prend la balle) Et je suis sorti. Et j'ai fermé la porte en silence. Et c'était comme si j'avais soudainement grandi.» 
noticia.html?ref=https:\%2F\%2Fwww.google.com\%2F>, consulté le 02/12/2020.

Caro. L, 2019b, «El número de inmigrantes menores no acompañados se duplica en 15 meses», ABC.ES, 15/07/2019, < https://www.abc.es/espana/abci-numeroinmigrantes-menores-no-acompanados-duplica-15-meses-201907140140_ noticia.html?ref=https:\%2F\%2Fwww.google.com\%2F>, consulté le $12 / 02 / 2020$.

Correa, I., 2011, Friday, Madrid, Sgae.

Doll, E-J, 2013, Los inmigrantes en la escena española contemporánea: Buscando una nueva identidad española. Madrid, Fundamento García Saleh, A., 2010, «Irma Correa obtiene el premio SGAE de teatro», in La Provincia, diario de las Palmas, 03/11/2010, <https://www.laprovincia.es/cultura/2010/11/03/irmacorrea-obtiene-premio-sgae-teatro/331898.html>, consulté le 02/02/2020.

Fiscal.es, 2018, <https:/www.fiscal.es/web/fiscal/-/extranjeriamemoria2018/ FISCALIA_SITE/recursos/pdf/capitulo_III/cap_III_4_7.pdf>, consulté le $13 / 03 / 2020$.

Golopentia S., 1994, Martinez M, Voir les didascalies. Toulouse/Paris, Cric/ Ophrys.

Pardellas J-M, 2007, «El niño polizón que sueña con Messi», El país, 17/11/2007, $<$ https://elpais.com/hemeroteca/elpais/portadas/2007/11/17/>, consulté le 05/12/2020.

Pavis P., 2004, Dictionnaire du théâtre, Armand Colin, Paris.

Soriano-Miras.R, 2008, “Análisis sociológico de la Ley 4/2000, de 11 de enero, sobre derechos y libertades de los extranjeros en España y su integración social. Sus reformas $(8 / 2000,11 / 2003,14 / 2003,2 / 2009)$ y su implicación social”, in Papers, Revista de Sociología, abril 2010, 96/3, <https://core.ac.uk/ download/pdf/39001361.pdf>, p. 692-693, consulté le 12/02/2020,.

Tornero, H., 2008, Sumergirse en el agua, Madrid, Sgae, Anaya.

Trésor de la langue Française, <https://www.cnrtl.fr/definition/ alt\%C3\%A9rit\%C3\%A9/substantif>, consulté le 16/08/2020.

Val E., 2001, «Conmoción en Alemania al reabrirse el caso del asesinato racista de un niño en 1997», El País, 25/11/2001.

Vuillaume, M., 2019, «Ici et là-bas à l'épreuve de la fiction», Scolia, 33, <https:// doi.org/10.4000/scolia.817>, consulté le 11/12/2020. 